\title{
Error Rates of M-PAM and M-QAM in Generalized Fading and Generalized Gaussian Noise Environments
}

\author{
Hamza Soury, Student Member, IEEE, Ferkan Yilmaz, Member, IEEE, \\ and Mohamed-Slim Alouini, Fellow, IEEE
}

\begin{abstract}
This letter investigates the average symbol error probability (ASEP) of pulse amplitude modulation and quadrature amplitude modulation coherent signaling over flat fading channels subject to additive white generalized Gaussian noise. The new ASEP results are derived in a generic closed-form in terms of the Fox $\mathrm{H}$ function and the bivariate Fox $\mathrm{H}$ function for the extended generalized-K fading case. The utility of this new general closed-form is that it includes some special fading distributions, like the Generalized-K, Nakagami-m, and Rayleigh fading and special noise distributions such as Gaussian and Laplacian. Some of these special cases are also treated and are shown to yield simplified results.
\end{abstract}

\section{Index Terms}

Symbol error probability, pulse amplitude modulation, quadrature amplitude modulation, additive generalized Gaussian noise, Laplacian noise, generalized-K fading, and Nakagami- $m$ fading.

\section{INTRODUCTION}

In pulse amplitude modulation (PAM), the information is conveyed by the amplitude of the pulse, and from two independent pulse amplitude modulation (PAM) signals (in-phase and quadrature PAM), one can easily generate any general order, and rectangular quadrature amplitude modulation (QAM) constellation. The average symbol error probability of PAM and QAM was extensively studied in the presence of a Gaussian noise and flat fading [1]-[5]. Although the Gaussian noise is widely used, the actual additive noise may deviate from it, so a more general noise model is sometimes needed. In fact, it has been widely reported that the generalized Gaussian distribution (GGD) can model different type of noise (see Table I), for example the Gaussian, Laplacian, and uniform distributions are just a special cases of the GGD [6], [7].

In the derivation of the ASEP of QAM in the standard Gaussian case, the challenge consists of solving an integral involving the product of two traditional Gaussian $Q$ functions [1]-[4]. Here, in the presence of generalized Gaussian noise, the problem is more complicated because we have to derive an integral of the product of two generalized Gaussian $Q$ functions [8]. This integral appears when we consider the ASEP of QAM over Extended Generalized-K (EGK) fading [9] and subject to additive white generalized Gaussian noise (AWGGN) [8]. In the PAM case, the problem is less complex than the QAM because we need to evaluate an integral involving one generalized Gaussian $Q$ function which is discussed in [8]. The EGK distribution has five parameters and includes most of the well-known fading distributions in the literature as either special or limit cases as shown in [9, Table 1] and Table II. Our goal in this letter to extend the analysis in [8] and [10], which were limited to binary coherent signalling and square $M$-QAM, respectively, and obtain a generic expression for the ASEP for $M$-PAM and $M$-QAM constellations and then to simplify these expressions as much as possible for some special cases of interest corresponding to different values of the fading and noise parameters.

H. Soury, F. Yilmaz, and M.-S. Alouini are with King Abdullah University of Science and Technology (KAUST), Thuwal, Makkah Province, Kingdom of Saudi Arabia (e-mail: soury.hamza, ferkan.yilmaz, slim.alouini\}@ @aust.edu.sa). 


\section{ASEP OF $M$-PAM AND $M$-QAM}

A typical single input single output communication system is considered in this letter. The transmitted signal $\mathcal{S}$ is mapped according to an $M$-PAM or $M$-QAM constellation. In fact, the QAM constellation is formed by two independent quadrature $M$-ary PAM signals, where the in-phase and quadrature signals are $M_{I}$-ary PAM and $M_{Q}$-ary PAM, respectively, and $M=M_{I} M_{Q}$. Next, $\mathcal{S}$ is multiplied by a channel fading envelope $\mathcal{H}$ that has a generalized flat fading characteristics and the resulting signal is then corrupted at the receiver end by an AWGGN $\mathcal{N}$ with zero mean, variance $\sigma^{2}$, and shaping parameter $\alpha$ (i.e. $\mathcal{R}=\mathcal{H} \mathcal{S}+\mathcal{N}$ ).

In [1] (respectively [5]), the probability of error of the system above is well studied for the Gaussian noise case, and the resulting symbol error probability (SEP) was shown to be given by [1, Eq. (10)] for the QAM constellation (respectively [5, Eq. (8.3.5)] for the PAM constellation).

Since the generalized generalized Gaussian distribution and the Gaussian distribution have the same symmetry properties, the SEP of the $M$-PAM signalling over an AWGGN channel can be easily written as

$$
P_{P A M}(E)=2\left(1-\frac{1}{M}\right) Q_{\alpha}\left(\frac{d}{\sigma}\right)
$$

where $d$ is the decision distance. Using the same symmetry properties, the SEP of the $M$-QAM can be written as

$$
\begin{gathered}
P_{Q A M}(E)=2\left(1-\frac{1}{M_{I}}\right) Q_{\alpha}\left(A_{I}\right)+2\left(1-\frac{1}{M_{Q}}\right) Q_{\alpha}\left(A_{Q}\right) \\
-4\left(1-\frac{1}{M_{I}}\right)\left(1-\frac{1}{M_{Q}}\right) Q_{\alpha}\left(A_{I}\right) Q_{\alpha}\left(A_{Q}\right)
\end{gathered}
$$

where $A_{I}=\frac{d_{I}}{\sigma}, A_{Q}=\frac{d_{Q}}{\sigma}, d_{I}$ and $d_{Q}$ are the in-phase and quadrature decision distances, respectively. In (1) and (2), $Q_{\alpha}($.$) is the generalized Gaussian Q$ function, defined defined, for $x \geq 0$, in [8, Eq. (A1)] as

$$
Q_{\alpha}(x)=\frac{\alpha \Lambda_{0}}{2 \Gamma(1 / \alpha)} \int_{x}^{\infty} \exp \left(-\Lambda_{0}^{\alpha} t^{\alpha}\right) d t
$$

where $\Gamma($.$) is the Gamma function and \Lambda_{0}=\sqrt{\Gamma(3 / \alpha) / \Gamma(1 / \alpha)}$, the absolute $\left(|t|^{\alpha}\right)$ is omitted from [8, Eq. (A1)] because we deal with positive parameters $(x \geq 0)$. The results in (1) and (2) can be found directly.

Before proceeding to the proof we have to compute the Bit error probability (BEP) of the BPSK constellation which is given in [8] as

$$
P_{B P S K}=Q_{\alpha}\left(\frac{d}{\sigma}\right)
$$

where $d$ is the decision distance and $\sigma^{2}$ is the noise variance.

It appears from [8] that the decision region is based on the euclidian distance between the received signal and the constellation symbols. We focus on computing the SEP of an $M$-PAM constellation. Consider an $M$-PAM constellation with decision distance $d$. The probability of error for the PAM is divided into two terms, one for the outermost points ( 2 points) and the other for the remaining $M-2$ points. The first term is similar for both points and is equal to the error between one outermost point and its closest neighbor which is equal to the error of BPSK with decision distance $d$ so $P_{\text {outer }}=\frac{1}{M} Q_{\alpha}\left(\frac{d}{\sigma}\right)$. In the other hand, each of the remaining $M-2$ points has two neighbors from both sided, so the probability of error is twice the probability of error between two symbols $P_{\text {inter }}=\frac{2}{M} Q_{\alpha}\left(\frac{d}{\sigma}\right)$. Hence to total probability of error of the $M$-PAM is given by

$$
P_{P A M}(E)=\underbrace{2 \frac{1}{M} Q_{\alpha}\left(\frac{d}{\sigma}\right)}_{\text {Two outermost points }}+\underbrace{2(M-2) \frac{1}{M} Q_{\alpha}\left(\frac{d}{\sigma}\right)}_{\text {The remaining } M-2 \text { points }}=2\left(1-\frac{1}{M}\right) Q_{\alpha}\left(\frac{d}{\sigma}\right) .
$$


Back to QAM model, the SEPs of the in-phase $M_{I}$-PAM and the quadrature $M_{Q}$-PAM are given by

$$
\begin{gathered}
P_{I}=2\left(1-\frac{1}{M_{I}}\right) Q_{\alpha}\left(\frac{d_{I}}{\sigma}\right), \\
P_{Q}=2\left(1-\frac{1}{M_{Q}}\right) Q_{\alpha}\left(\frac{d_{Q}}{\sigma}\right) .
\end{gathered}
$$

A correct reception appears when we have correct reception in the independent in-phase signals and the quadrature phase signals. Then the probability of correct symbol reception for the rectangular $M$-QAM system is

$$
P_{c}=\left(1-P_{I}\right)\left(1-P_{Q}\right),
$$

and the SEP is given by

$$
P_{S}=1-P c=1-\left(1-P_{I}\right)\left(1-P_{Q}\right)=P_{I}+P_{Q}-P_{I} P_{Q} .
$$

Using (6) and (7) in (8), we get

$$
P_{S}=2\left(1-\frac{1}{M_{I}}\right) Q_{\alpha}\left(A_{I}\right)+2\left(1-\frac{1}{M_{Q}}\right) Q_{\alpha}\left(A_{Q}\right)-4\left(1-\frac{1}{M_{I}}\right)\left(1-\frac{1}{M_{Q}}\right) Q_{\alpha}\left(A_{I}\right) Q_{\alpha}\left(A_{Q}\right) .
$$

In the presence of a slow fading channel, the ASEP is given by averaging the conditional SEPs (1) and (2) over the probability density function (PDF) of the fading power $\gamma=\mathcal{H}^{2}, p_{\gamma}(\gamma)$, yielding

$$
\begin{aligned}
& \operatorname{Pr}_{P A M}(e)=2\left(1-\frac{1}{M}\right) \mathcal{I}\left(\frac{d}{\sigma}\right), \\
& \operatorname{Pr}_{Q A M}(e)=2\left(1-\frac{1}{M_{I}}\right) \mathcal{I}\left(A_{I}\right)+2\left(1-\frac{1}{M_{Q}}\right) \mathcal{I}\left(A_{Q}\right)-4\left(1-\frac{1}{M_{I}}\right)\left(1-\frac{1}{M_{Q}}\right) \mathcal{J},
\end{aligned}
$$

where we define

$$
\begin{aligned}
\mathcal{I}(x) & =\int_{0}^{\infty} Q_{\alpha}(\sqrt{\gamma} x) p_{\gamma}(\gamma) d \gamma \\
\mathcal{J} & =\int_{0}^{\infty} Q_{\alpha}\left(\sqrt{\gamma} A_{I}\right) Q_{\alpha}\left(\sqrt{\gamma} A_{Q}\right) p_{\gamma}(\gamma) d \gamma
\end{aligned}
$$

with $x$ taking the values $A_{I}, A_{Q}$, or $\frac{d}{\sigma}$ in (11).

\section{AVERAGE SEP OVER EGK FADING}

We consider an EGK fading environment [9] for which $\gamma$ follows an EGK PDF [9, Eq. (2)], with average fading power $\bar{\gamma}=\mathbb{E}[\gamma]$, fading severity figure $m \geq 0$, fading shaping factor $\xi \geq 0$, shadowing severity figure $m_{s} \geq \frac{1}{2}$, and shadowing shaping factor $\xi_{s} \geq 0$

$$
p_{\gamma}(\gamma)=\frac{\xi}{\Gamma\left(m_{s}\right) \Gamma(m)}\left(\frac{\beta_{s} \beta}{\bar{\gamma}}\right)^{m \xi} \gamma^{m \xi-1} \Gamma\left(m_{s}-m \frac{\xi}{\xi_{s}}, 0,\left(\frac{\beta_{s} \beta}{\bar{\gamma}}\right)^{m \xi} \gamma^{\xi}, \frac{\xi}{\xi_{s}}\right),
$$

where $\beta=\Gamma(m+1 / \xi) / \Gamma(m), \beta_{s}=\Gamma\left(m_{s}+1 / \xi_{s}\right) / \Gamma\left(m_{s}\right)$, and $\Gamma(\cdot, \cdot, \cdot, \cdot)$ is the extended incomplete Gamma function defined in [11, Eq. (6.2)] as

$$
\Gamma(a, x, b, c)=\int_{x}^{\infty} r^{a-1} \exp \left(-r-b r^{-c}\right) d r,
$$

where $a, b, c \in \mathbb{C}$ and $x \in \mathbb{R}^{+}$

As $\mathcal{I}$ and $\mathcal{J}$ are difficult to manipulate using the classical expressions of the EGK distribution and the GGD, we use for alternative expressions of $Q_{\alpha}($.$) as given in (3) and p_{\gamma}(\gamma)$ as defined in [9, Eq. (2)], in order to derive closed-from expressions for $\mathcal{I}$ and $\mathcal{J}$ and as a result for the ASEP. More specifically, as 
was done in [8], we first express (3) and [9, Eq. (2)] in terms of the Fox H function (FHF) H. [.] [12, Eq. (1.1.1)], whose MATHEMATICA implementation is given in [13, Appendix]. As such $Q_{\alpha}($.$) and p_{\gamma}(\gamma)$ are expressed in terms of the FHF in [8, Eq. (A.5)] and [8, Eq. (8)] respectively

$$
\begin{gathered}
Q_{\alpha}(x)=\frac{1}{2 \Gamma(1 / \alpha)} \mathrm{H}_{1,2}^{2,0}\left[\Lambda_{0}^{\alpha}|x|^{\alpha} \mid \begin{array}{c}
(1,1) \\
\left(\frac{1}{\alpha}, 1\right),(0,1)
\end{array}\right], \\
p_{\gamma}(\gamma)=\frac{1}{\Gamma\left(m_{s}\right) \Gamma(m) \gamma} \mathrm{H}_{0,2}^{2,0}\left[\frac{\beta_{s} \beta}{\bar{\gamma}} \gamma \mid \begin{array}{c}
\left(m_{s}, \frac{1}{\xi_{s}}\right),\left(m, \frac{1}{\xi}\right)
\end{array}\right],
\end{gathered}
$$

The FHF is defined in [12, Eq. (1.1.1)] as

$$
\mathrm{H}_{p, q}^{m, n}\left[z \mid \begin{array}{l}
\left(a_{i}, \alpha_{i}\right)_{1, p} \\
\left(b_{j}, \beta_{j}\right)_{1, q}
\end{array}\right]=\frac{1}{2 \pi i} \int_{\mathcal{C}} \frac{\prod_{j=1}^{m} \Gamma\left(b_{j}+\beta_{j} s\right) \prod_{i=1}^{n} \Gamma\left(1-a_{i}-\alpha_{i} s\right)}{\prod_{j=n+1}^{p} \Gamma\left(a_{i}+\alpha_{i} s\right) \prod_{j=m+1}^{q} \Gamma\left(1-b_{j}-\beta_{j} s\right)} z^{-s} d s,
$$

In (11), $\mathcal{I}(x)$ includes an integral involving the product of two FHFs which is similar to what the authors studied in [8]. Following similar steps to the ones adopted in [8], the identity [12, Eq. (2.8.4)] was very helpful to find a closed form of $\mathcal{I}($.)

$$
\begin{aligned}
& \int_{0}^{\infty} t^{\eta-1} \mathrm{H}_{p, q}^{m, n}\left[z t^{\sigma} \mid \begin{array}{c}
\left(a_{i}, \alpha_{i}\right)_{1, p} \\
\left(b_{j}, \beta_{j}\right)_{1, q}
\end{array}\right] \mathrm{H}_{P, Q}^{M, N}\left[w t \mid \begin{array}{c}
\left(c_{i}, \gamma_{i}\right)_{1, P} \\
\left(d_{j}, \delta_{j}\right)_{1, Q}
\end{array}\right] d t \\
&=\quad w^{-\eta} \mathrm{H}_{p+Q, q+P}^{m+N, n+M}
\end{aligned}\left[\begin{array}{ll}
z w^{-\sigma} & \left.\begin{array}{c}
\left(a_{i}, \alpha_{i}\right)_{1, n},\left(1-d_{j}-\eta \delta_{j}, \sigma \delta_{j}\right)_{1, Q},\left(a_{i}, \alpha_{i}\right)_{n+1, p} \\
\left(b_{j}, \beta_{j}\right)_{1, m},\left(1-c_{j}-\eta \gamma_{j}, \sigma \gamma_{j}\right)_{1, P},\left(b_{j}, \beta_{j}\right)_{m+1, q}
\end{array}\right] .
\end{array}\right.
$$

and a closed form for $\mathcal{I}($.$) in terms of the FHF is easily found as$

$$
\mathcal{I}(x)=\frac{\mathrm{H}_{2,3}^{2,2}\left[\frac{\beta_{s} \beta}{x^{2} \Lambda_{0}^{2} \bar{\gamma}} \mid \begin{array}{c}
\left(1-\frac{1}{\alpha}, \frac{2}{\alpha}\right),\left(1, \frac{2}{\alpha}\right) \\
\left(m_{s}, \frac{1}{\xi_{s}}\right),\left(m, \frac{1}{\xi}\right),\left(0, \frac{2}{\alpha}\right)
\end{array}\right]}{\alpha \Gamma(1 / \alpha) \Gamma\left(m_{s}\right) \Gamma(m)} .
$$

Now substituting (15) and (16) in (12), $\mathcal{J}$ is defined as an integral involving the product of three FHFs. Using the identity [14, Eq. (2.3)]

$$
\begin{aligned}
\int_{0}^{\infty} x^{\lambda-1} \mathrm{H}_{p, q}^{m, 0}\left[a x \mid \begin{array}{l}
\left(a_{i}, \alpha_{i}\right)_{1, p} \\
\left(b_{j}, \beta_{j}\right)_{1, q}
\end{array}\right] \mathrm{H}_{p_{2}, q_{2}}^{m_{2}, n_{2}}\left[\beta x^{h} \mid \begin{array}{c}
\left(c_{i}, r_{i}\right)_{1, p_{2}} \\
\left(d_{j}, \delta_{j}\right)_{1, q_{2}}
\end{array}\right] \mathrm{H}_{p_{3}, q_{3}}^{m_{3}, n_{3}}\left[\delta x^{k} \mid \begin{array}{l}
\left(e_{i}, E_{i}\right)_{1, p_{3}} \\
\left(f_{j}, F_{j}\right)_{1, q_{3}}
\end{array}\right] d x \\
=a^{-\lambda} \mathrm{H}_{p, q ; p_{2}, q_{2} ; p_{3}, q_{3}}^{0, m ; m_{2}, n_{2} ; m_{3}, n_{3}}\left[\begin{array}{c}
\left(1-b_{j}-\lambda \beta_{j} ; h \beta_{j}, k \beta_{j}\right)_{1, q} \\
\left(1-a_{i}-\lambda \alpha_{i} ; h \alpha_{i}, k \alpha_{i}\right)_{1, p} \\
\left(c_{i}, r_{i}\right)_{1, p_{2}} \\
\left(d_{j}, \delta_{j}\right)_{1, q_{2}} \\
\left(e_{i}, E_{i}\right)_{1, p_{3}} \\
\left(f_{j}, F_{j}\right)_{1, q_{3}}
\end{array}\left|\frac{\beta}{a^{h}, \frac{\delta}{a^{k}}}\right|,\right.
\end{aligned}
$$

$\mathcal{J}$ can be expressed in terms of the FHF of two variables [14], also known as the Bivariate Fox H-function (BFHF) $\mathrm{H} . . . \ldots \ldots . . . . . .,$.$] and whose MATLAB implementation is outlined in [15]. Hence a closed-form for \mathcal{J}$ is found as

$$
\begin{array}{r}
\mathcal{J}=\frac{1}{2 \alpha \Gamma(1 / \alpha)^{2} \Gamma\left(m_{s}\right) \Gamma(m)} \\
\mathrm{H}_{2,1 ; 0,2 ; 1,2}^{0,2 ; 2,2,0}\left[\begin{array}{c}
\left(1-\frac{1}{\alpha} ; \frac{2}{\alpha}, 1\right),\left(1 ; \frac{2}{\alpha}, 1\right) \\
\left(0 ; \frac{2}{\alpha}, 1\right) \\
\left(m_{s}, \frac{1}{\xi_{s}}\right),\left(m, \frac{1}{\xi}\right) \\
(1,1) \\
\left(\frac{1}{\alpha}, 1\right),(0,1)
\end{array} \mid \frac{\beta_{s} \beta}{A_{I}^{2} \Lambda_{0}^{2} \bar{\gamma}},\left(\frac{A_{Q}}{A_{I}}\right)^{\alpha}\right.
\end{array} .
$$


In conclusion, a closed-form of the ASEP of QAM (respectively PAM) is obtained using (19) and (21) in (10) (respectively (19) in (9)). This closed-form is a general expression of the ASEP of rectangular $\left(M_{I} \neq M_{Q}\right)$, square $\left(M_{I}=M_{Q}\right)$ QAM, and PAM in arbitrary EGK fading subject to AWGGN, and holds as such for a considerable range of noise and fading parameters.

\section{Special Cases of Noise And Fading}

\section{A. EGK Fading with Additive Laplacian Noise}

The first special case of the generalized Gaussian noise appears when $\alpha$ equals to 1 (i.e. the noise is Laplacian). Using the properties and the special cases of the FHF [12], and BFHF functions [14], the general ASEP expression in (10) and (9) can be simplified by re-writing $\mathcal{I}($.$) and \mathcal{J}$ as

$$
\begin{aligned}
\mathcal{I}(x) & =\frac{1}{\Gamma\left(m_{s}\right) \Gamma(m)} \mathrm{H}_{1,2}^{2,1}\left[\frac{\beta_{s} \beta}{2 x^{2} \bar{\gamma}} \mid \begin{array}{c}
(1,2) \\
\left(m_{s}, \frac{1}{\xi_{s}}\right),\left(m, \frac{1}{\xi}\right)
\end{array}\right] \\
\mathcal{J} & \left.=\frac{1}{2 \Gamma\left(m_{s}\right) \Gamma(m)} \mathrm{H}_{1,0 ; 0,2 ; 0,1}^{0,1 ; 2,0 ; 1,0}\left[\begin{array}{c}
(1 ; 2,1) \\
\left(m_{s}, \frac{1}{\left.\xi_{s}\right),(m}, \frac{1}{\xi}\right)
\end{array}\right] \frac{\beta_{s} \beta}{2 A_{I}^{2} \bar{\gamma}}, \frac{A_{Q}}{A_{I}}\right] .
\end{aligned}
$$

In fact, $\mathcal{J}$ can be expressed in terms of $\mathcal{I}($.$) for the Laplacian noise (LN). Indeed using the definition of$ BHFH [14, Eq. (1.1)], we re-write $\mathcal{J}$ as

$$
\mathcal{J}=\frac{1}{2 \Gamma\left(m_{s}\right) \Gamma(m)} \frac{1}{(2 \pi i)^{2}} \int_{L_{1}} \int_{L_{2}} \Gamma(-2 s-t) \Gamma\left(m_{s}+s / \xi_{s}\right) \Gamma(m+s / \xi) \Gamma(t)\left(\frac{\beta_{s} \beta}{2 A_{I}^{2} \bar{\gamma}}\right)^{-s}\left(\frac{A_{Q}}{A_{I}}\right)^{-t} d t d s
$$

Using the inverse Mellin transform of the extended incomplete Gamma function $\Gamma(., . ; . ;$.$) [11, Eq. (6.29)],$ and its special case presented in [11, Eq. (6.42)], we get

$$
\begin{aligned}
\frac{1}{2 \pi i} \int_{L_{2}} \Gamma(-2 s-t) \Gamma(t)\left(\frac{A_{Q}}{A_{I}}\right)^{-t} d t & =\Gamma\left(-2 s, 0 ; \frac{A_{Q}}{A_{I}} ;-1\right) \\
& =\Gamma(-2 s)\left(1+\frac{A_{Q}}{A_{I}}\right)^{2 s} .
\end{aligned}
$$

Replacing (25) in (24)

$$
\begin{aligned}
\mathcal{J} & =\frac{1}{2 \Gamma\left(m_{s}\right) \Gamma(m)} \frac{1}{2 \pi i} \int_{L_{1}} \Gamma\left(m_{s}+s / \xi_{s}\right) \Gamma(m+s / \xi) \Gamma(-2 s)\left(\frac{\beta_{s} \beta}{2\left(A_{I}+A_{Q}\right)^{2} \bar{\gamma}}\right)^{-s} d s \\
& =\frac{1}{2 \Gamma\left(m_{s}\right) \Gamma(m)} \mathrm{H}_{1,2}^{2,1}\left[\frac{\beta_{s} \beta}{2\left(A_{I}+A_{Q}\right)^{2} \bar{\gamma}} \mid \begin{array}{c}
(1,2) \\
\left(m_{s}, \frac{1}{\xi_{s}}\right),\left(m, \frac{1}{\xi}\right)
\end{array}\right] \\
& =\frac{1}{2} \mathcal{I}\left(A_{I}+A_{Q}\right) .
\end{aligned}
$$

Hence, in the presence of LN, the ASEP is defined in terms of $\mathcal{I}($.$) only. The expression (22) contains$ also the FHF but with a lower rank than the general expressions given in (19), which means that it can be computed with a reduced complexity of evaluation. Since the LN is an interesting case of study, it is also of interest to study other special fading cases in conjunction with the LN. For instance, considering the generalized Nakagami-m (GNM) fading by setting $\xi_{s}=1$ and $m_{s} \rightarrow \infty$ in (22), $\mathcal{I}($.$) further simplifies to$

$$
\mathcal{I}(x)=\frac{1}{\Gamma(m)} \mathrm{H}_{1,1}^{1,1}\left[\frac{\beta}{2 x^{2} \bar{\gamma}} \mid \begin{array}{c}
(1,2) \\
\left(m, \frac{1}{\xi}\right)
\end{array}\right] \text {. }
$$


Re-writing the expression of the FHF, using some changes of variable, and the identity [11, Eq. (6.29)], a new expression of $\mathcal{I}($.$) can be obtained as$

$$
\mathcal{I}(x)=\frac{\xi}{\Gamma(m)}\left(\frac{\beta}{2 x^{2} \bar{\gamma}}\right)^{m \xi} \Gamma\left(2 m \xi, 0 ;\left(\frac{\beta}{2 x^{2} \bar{\gamma}}\right)^{m \xi} ;-2 \xi\right) .
$$

\section{B. Rayleigh Fading with Additive Laplacian Noise}

Keeping an additive LN, the Rayleigh fading case is obtained by setting $m=1$ and $\xi=1$ in (28). From (28) and with a simple manipulation of $\Gamma(., . ; . ;$.$) , it can be shown that \mathcal{I}$ is equivalent to

$$
\begin{aligned}
\mathcal{I}(x) & =\frac{1}{2 x^{2} \bar{\gamma}} \Gamma\left(2,0 ; \frac{1}{2 x^{2} \bar{\gamma}} ;-2\right) \\
& =\frac{1}{2 x^{2} \bar{\gamma}} \int_{0}^{\infty} t e^{-t-\frac{t^{2}}{2 x^{2} \bar{\gamma}}} d t \\
& =\frac{e^{\frac{x^{2} \bar{\gamma}}{2}}}{\sqrt{2 x^{2} \bar{\gamma}}} \int_{\sqrt{\frac{x^{2} \bar{\gamma}}{2}}}^{\infty}\left(\sqrt{2 x^{2} \bar{\gamma}} u-x^{2} \bar{\gamma}\right) e^{-u^{2}} d u \text { where } u=\frac{t}{\sqrt{2 x^{2} \bar{\gamma}}}+\frac{\sqrt{2 x^{2} \bar{\gamma}}}{2} \\
& =e^{\frac{x^{2} \bar{\gamma}}{2}} \int_{\sqrt{\frac{x^{2} \bar{\gamma}}{2}}}^{\infty} u e^{-u^{2}} d u-\frac{e^{\frac{x^{2} \bar{\gamma}}{2}} \sqrt{2 x^{2} \bar{\gamma}}}{2} \int_{\sqrt{\frac{x^{2} \bar{\gamma}}{2}}}^{\infty} e^{-u^{2}} d u \\
& =\frac{1}{2}-\frac{x}{2} \sqrt{2 \pi \bar{\gamma}} e^{\frac{\bar{\gamma}^{2}}{2}} Q(x \sqrt{\bar{\gamma}})
\end{aligned}
$$

which is a very simple expression involving only the standard Gaussian $Q$ function (i.e. $Q(x)=Q_{2}(x)$ ). To the best of the author's knowledge, the expressions of the ASEP with additive LN and Rayleigh fading in (29) is a new expression that has not been reported previously.

\section{Generalized K (GK) Fading with Additive Gaussian Noise}

The last two special cases will focus on the classical Gaussian noise with different fading distributions. The first example deals with the performance of $M$-PAM and $M$-QAM over a GK fading subjected to an additive white gaussian noise (AWGN) channel. This case is obtained by setting $\alpha=2, \xi=1$, and $\xi_{s}=1$. With these settings, the main integrals in (19) and (21) reduce to

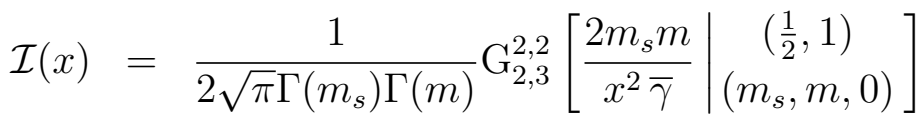

$$
\begin{aligned}
& \mathcal{J}=\frac{1}{4 \pi \Gamma\left(m_{s}\right) \Gamma(m)}
\end{aligned}
$$

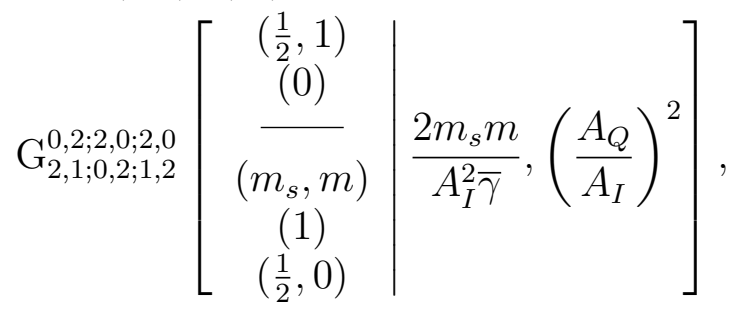

where $\mathcal{I}$ is expressed in terms of the standard Meijer G-function (MGF) [12, Eq. (2.9.1)] which is a built in function in MATHEMATICA, and $\mathcal{J}$ is expressed in terms of the bivariate Meijer G-function (BMGF) whose MATHEMATICA implementation is given in [16, Table II].

It is necessary to mention that in these special cases, we are not just setting the parameters in the general expression (19) and (21). Rather, in each case, we offered simplified closed-form expressions of the main parts of the ASEP with reduced computational complexity. In fact, from the definition of the FHF [12, Eq.(1.1.1)], the complexity of evaluating the Mellin integral is based on the rank of the FHF, which is reduced in these special cases. 


\section{Rayleigh Fading with Additive Gaussian Noise}

The AWGN was studied in the second special case and an expression of the ASEP of $M$-PAM and $M$-QAM over GK fading with AWGN was derived and can be obtained by (30) and (31). The Rayleigh fading is a special case of the GK distribution by simply setting $m=1$ and $m_{s} \rightarrow \infty$. In this case, the main integrals, $\mathcal{I}$ and $\mathcal{J}$ in (30) and (31), respectively reduce to

$$
\begin{aligned}
\mathcal{I}(x)=\frac{1}{2 \sqrt{\pi}} \mathrm{G}_{2,2}^{1,2}\left[\frac{2}{x^{2} \bar{\gamma}} \mid \begin{array}{c}
\left(\frac{1}{2}, 1\right) \\
(1,0)
\end{array}\right] \\
\mathcal{J}=\frac{1}{4 \pi} \mathrm{G}_{2,1 ; 0,1 ; 1,2}^{0,2 ; 1,0 ; 2,0}\left[\begin{array}{c}
\left.\frac{1}{2}, 1\right) \\
\frac{(0)}{(1)} \\
(1) \\
\left(\frac{1}{2}, 0\right)
\end{array} \mid \frac{2}{A_{I}^{2} \bar{\gamma}},\left(\frac{A_{Q}}{A_{I}}\right)^{2}\right] .
\end{aligned}
$$

The expressions in (32) and (33) involve reduced rank MGF and BMGF functions in comparison with (30) and (31). However, it is interesting to note that these formulas are numerically equivalent to

$$
\begin{aligned}
\mathcal{I}(x) & =\frac{1}{2}-\frac{1}{2} \sqrt{\frac{x^{2} \bar{\gamma}}{2+x^{2} \bar{\gamma}}} \\
\mathcal{J} & =\frac{1}{4}-\frac{1}{2 \pi}\left[\sqrt{\frac{A_{I}^{2} \bar{\gamma}}{2+A_{I}^{2} \bar{\gamma}}} \tan ^{-1}\left(\frac{A_{I}}{A_{Q}} \sqrt{\frac{2+A_{I}^{2} \bar{\gamma}}{A_{I}^{2} \bar{\gamma}}}\right)\right. \\
& \left.+\sqrt{\frac{A_{Q}^{2} \bar{\gamma}}{2+A_{Q}^{2} \bar{\gamma}}} \tan ^{-1}\left(\frac{A_{Q}}{A_{I}} \sqrt{\frac{2+A_{Q}^{2} \bar{\gamma}}{A_{Q}^{2} \bar{\gamma}}}\right)\right],
\end{aligned}
$$

where $\tan ^{-1}($.$) is the inverse tangent function, in agreement with the known expressions of Beaulieu [1,$ Eq. (5)] of the ASEP of $M$-QAM over Rayleigh fading and subject to AWGN.

\section{Numerical RESUlts AND CONCLUding REMARKS}

Let us consider an $8 \times 4$-QAM system, as presented in [1], and investigate the system performance as a function of the signal-to-noise ratio (SNR) for different type of noise (i.e. different values of $\alpha$ ), and fading (i.e. different set of values of $m, m_{s}, \xi$, and $\xi_{s}$ ), proceeding of PAM performance is less complex than the QAM so we focus in this part on the QAM performance. Let us define the in-phase-to-quadrature decision distance ratio $\tau=d_{Q} / d_{I}=A_{Q} / A_{I}$. In this case the average total energy per symbol $E_{T}$ can be defined as $E_{T}=\frac{21}{2} \bar{\gamma} d_{I}^{2}+\frac{5}{2} \bar{\gamma} d_{Q}^{2}=0.5\left(21+5 \tau^{2}\right) \bar{\gamma} d_{I}^{2}$. According to this definition, the SNR has the following expression $\frac{E_{T}}{\sigma^{2}}=0.5\left(21+5 \tau^{2}\right) \bar{\gamma} A_{I}^{2}$. For instance, $\tau$ is fixed $\left(\tau=(21 / 5)^{1 / 5}\right)$, so the average energies of the in-phase and quadrature signals are equal.

The first studied case is a comparison between the Gaussian and Laplacian noises in conjunction with Nakagami- $m$ fading. Note that the Nakgami- $m$ fading is obtained by setting the fading shaping factor $\xi=1$ in (28) for the LN. Fig. 1 shows the ASEP as a function of the average SNR per symbol $\frac{E_{T}}{\sigma^{2}}$ for both cases Gaussian and Laplacian and for four values of the fading parameter, namely $m=\frac{1}{2}, 2,4, \infty$. A comparison between the analytical results, presented in this letter, and a computer-based Monte-Carlo simulations results are presented. The simulation results match perfectly the analytical results derived in this letter. A general look at the figure shows us that the performance of the system is improved by increasing the fading parameter $m$, as expected. In addition, for lower SNR (i.e. SNR $<15 \mathrm{~dB}$ ), we note that the ASEP of the LN is better than the ASEP of the Gaussian noise. The situation reverse for high SNR and low amount of fading $(m \geq 2)$ since the Gaussian noise yields better results than LN. However for high amount of fading, such as for example $m=1 / 2$, the performance in LN is better than in the Gaussian noise case.

In the second numerical example, we compare the Rayleigh fading and the Nakagami-4 fading. For instance, in Fig. 2, we draw the ASEP in function of the SNR per QAM symbol for different values of $\alpha$. Note again that the simulation results match perfectly the analytical results obtained from (19) and (21). 
In Rayleigh fading case, it is worth mentioning that the system has better performance by decreasing the noise parameter $\alpha$, which confirm the result found in the previous example when the LN had better performance than the Gaussian noise for high amount of fading. However, the situation becomes different for lower amount of fading (i.e. when $m=4$ ), and we get two regions. For low SNR the ASEP decreases with the noise parameter, and for high SNR it gets better by increasing $\alpha$.

A new parameter appears on the energy expression, which is $\tau$, and in this numerical example we want to see the effect of $\tau$ on the system performance. Therefore Fig. 3 draws the ASEP of the system described above as a function of the SNR and for different values of the in-phase-to-quadrature decision distance ratio. It is clear that the best case is when the in-phase and quadrature distance are equal (i.e. $\tau=1$ ) for both cases of noise. For equal energy between the in-phase and quadrature signal (i.e. $\tau=(21 / 5)^{1 / 2}$ ), the system looses in performance but in small amount (about $1 \mathrm{~dB}$ in SNR for large SNRs). However, the loss is more important when the quadrature signal has 21/5 times the average energy of the in-phase signal (i.e. $\tau=21 / 5$ ), since it incurs a loss of about $4 \mathrm{~dB}$ in SNR for large SNRs, relative to the case where $\tau=1$.

\section{REFERENCES}

[1] N. Beaulieu, "A useful integral for wireless communication theory and its application to rectangular signaling constellation error rates," IEEE Trans. Commun., vol. 54, no. 5, pp. 802-805, May 2006.

[2] G. Karagiannidis, "On the symbol error probability of general order rectangular QAM in Nakagami-m fading," IEEE Commun. Lett., vol. 10, no. 11, pp. 745-747, November 2006.

[3] R. Mallik, "Average of product of two Gaussian Q-functions and its application to performance analysis in Nakagami fading," IEEE Trans. Commun., vol. 56, no. 8, pp. 1289-1299, August 2008.

[4] H. Suraweera and J. Armstrong, "A simple and accurate approximation to the SEP of rectangular QAM in arbitrary Nakagami-m fading channels," IEEE Communications Letters, vol. 11, no. 5, pp. 426-428, May 2007.

[5] J. G. Proakis and M. Salehi, Communication Systems Engineering, 2nd ed. Upper Saddle River, NJ, USA: Prentice-Hall, August 2001.

[6] R. Viswanathan and A. Ansari, "Distributed detection of a signal in generalized Gaussian noise," IEEE Transcations on Acoustics, Speech and Signal Processing, vol. 37, no. 5, pp. 775-778, May 1989.

[7] S. Zahabi and A. Tadaion, "Local spectrum sensing in non-Gaussian noise," in Proc. of the IEEE 17th International Conference on Telecommunication (ICT'2010), Doha, Qatar, April 2010, pp. 843-847.

[8] H. Soury, F. Yilmaz, and M.-S. Alouini, "Average bit error probability of binary coherent signaling over generalized fading channels subject to additive generalized Gaussian noise," IEEE Commun. Lett., vol. 16, no. 6, pp. 785-788, June 2012.

[9] F. Yilmaz and M.-S. Alouini, "A new simple model for composite fading channels: Second order statistics and channel capacity," in Proc. of the IEEE 7th International Symposium on Wireless Communication Systems (ISWCS'2010), York, UK, Sept. 2010, pp. 676-680.

[10] H. Soury, F. Yilmaz, and M.-S. Alouini, "Exact symbol error probability of square M-QAM signaling over generalized fading channels subject to additive generalized Gaussian noise," in Proc. of the IEEE International Symposium on Information Theory (ISIT'2013), Istanbul, Turkey, July 2013.

[11] M. A. Chaudhry and S. M. Zubair, On a Class of Incomplete Gamma Function with Applications. Boca Raton-London-Ney YorkWashington, D.C.: Chapman \& Hall/CRC, 2002.

[12] A. Kilbas and M. Saigo, H-Transforms : Theory and Applications (Analytical Method and Special Function), 1st ed. CRC Press, 2004.

[13] F. Yilmaz and M.-S. Alouini, "Product of the powers of generalized Nakagami-m variates and performance of cascaded fading channels," in Proc. of the IEEE Global Telecommunication Conference (GLOBECOM'2009), Honolulu, Hawai, USA, Dec 2009, pp. 1-8.

[14] P. Mittal and K. Gupta, "An integral involving generalized function of two variables," in Indian Acad. Sci., 1972, pp. 117-123.

[15] K. Peppas, "A new formula for the average bit error probability of dual-hop amplify-and-forward relaying systems over generalized shadowed fading channels," IEEE Wireless Commun. Lett., vol. 1, no. 2, pp. 85-88, Apr 2012.

[16] I. Ansari, S. Al-Ahmadi, F. Yilmaz, M.-S. Alouini, and H. Yanikomeroglu, "A new formula for the BER of binary modulations with dual-branch selection over generalized-K composite fading channels," IEEE Trans. Commun., vol. 59, pp. 2654-2658, Oct 2011.

[17] E. Stacy, "A generalization of the gamma distribution," The Annals of Mathematical Statistics, vol. 3, no. 33, pp. 1187-1192, Sep. 1962.

[18] V. Aalo, T. Piboongungon, and C.-D. Iskander, "Bit-error rate of binary digital modulation schemes in generalized gamma fading channels," IEEE Communications Letters, vol. 9, no. 2, pp. 139-141, Feb. 2005.

[19] H. Suzuki, "A statistical model for urban radio propogation," IEEE Transactions on Communications, vol. 25, no. 7, pp. 673-680, July 1977.

[20] M. H. Ismail and M. M. Matalgah, "Outage probability in multiple access systems with weibull-faded lognormal-shadowed communication links," in in Proc of the IEEE 62nd Vehicular Technology Conference (VTC-2005-Fall), vol. 4, Dallas, TX, Sept. 2005, pp. 2129-2133. 


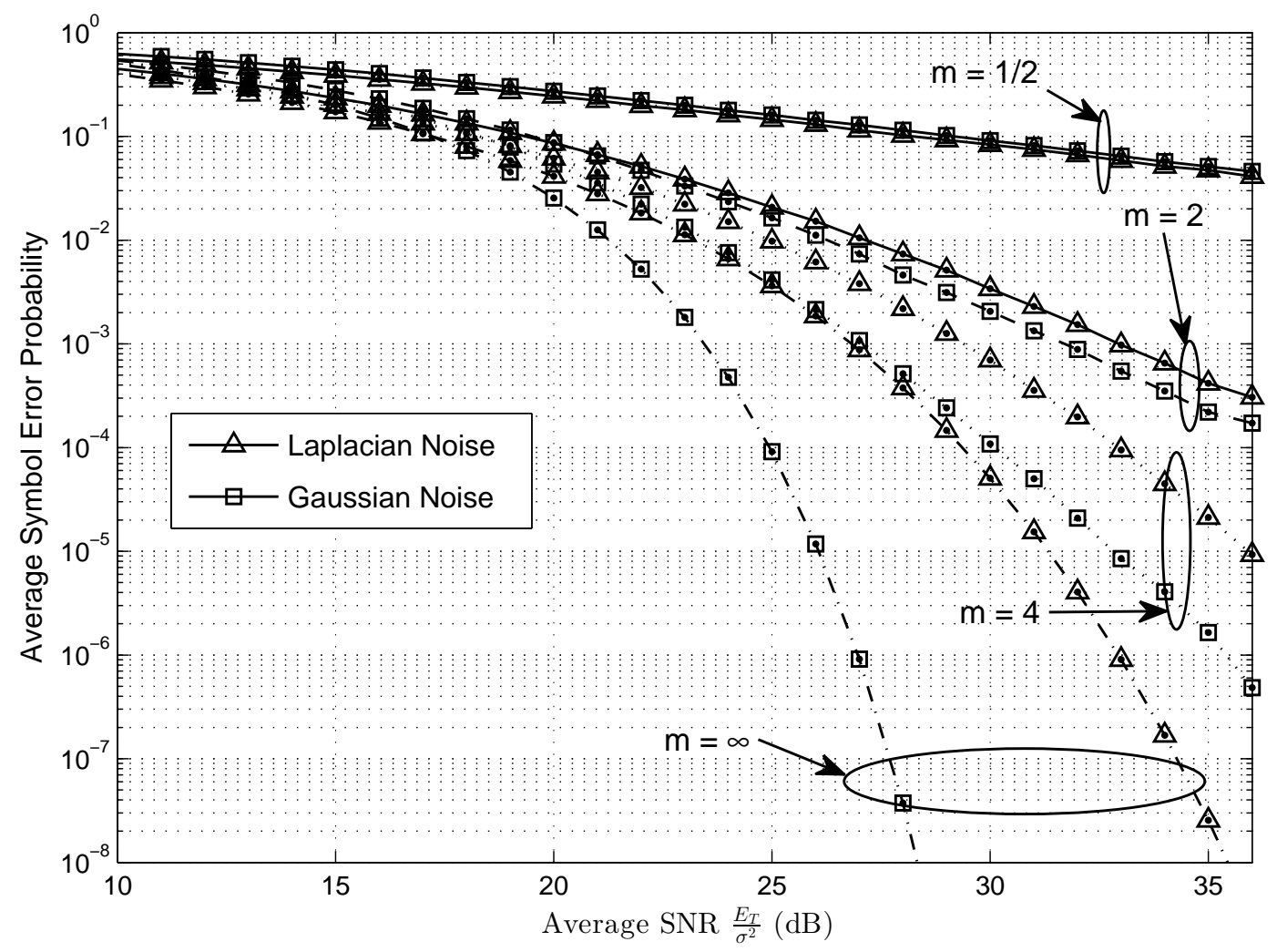

Fig. 1. ASEP of $8 \times 4$-QAM over Nakagami- $m$ fading subject to Laplacian noise and Gaussian noise. The markers denote simulation results while the lines represent analytical results. 


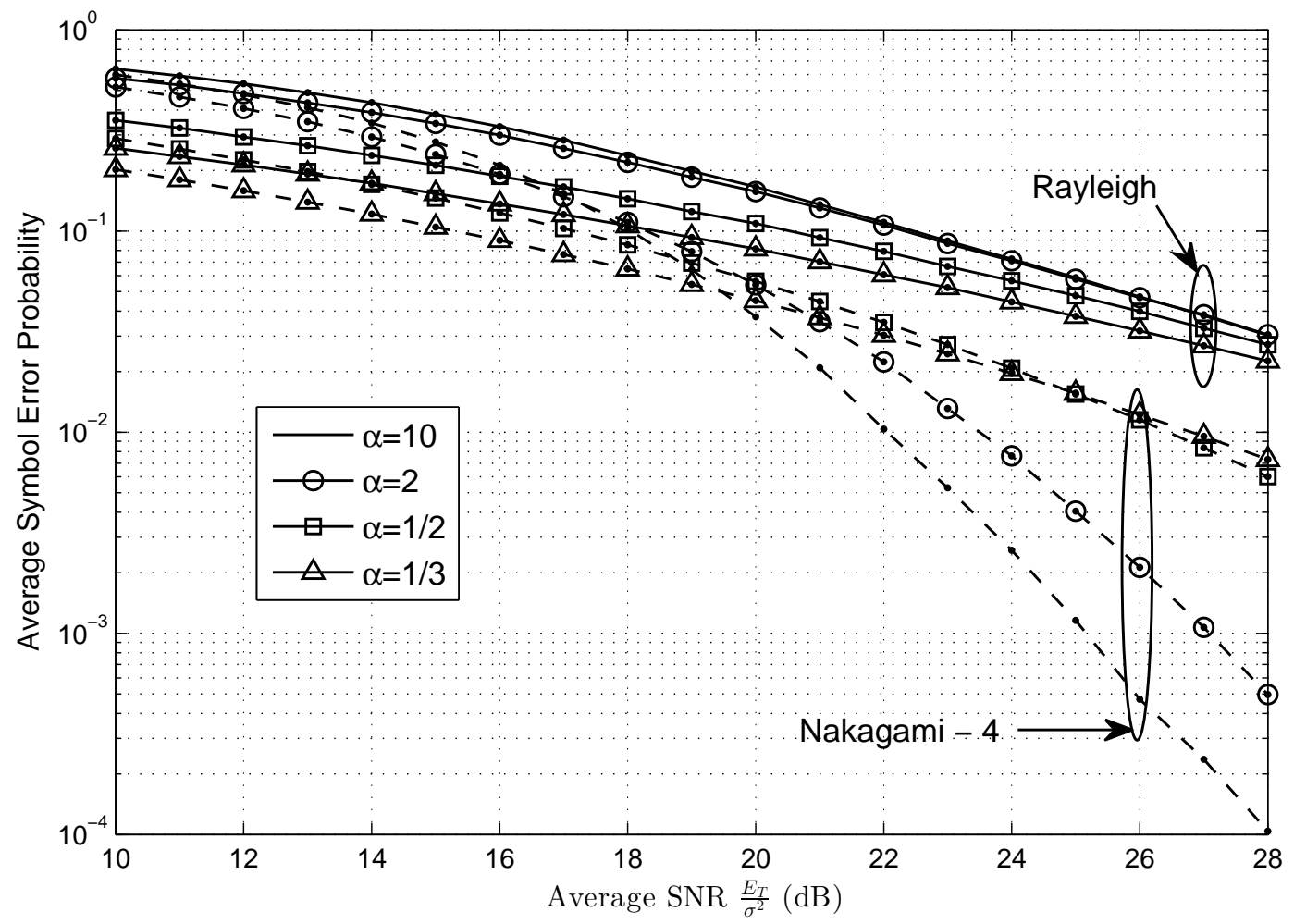

Fig. 2. ASEP of $8 \times 4$-QAM over Rayleigh and Nakagami-4 fading subject to generalized Gaussian noise. The markers denote simulation results while the lines represent analytical results. 


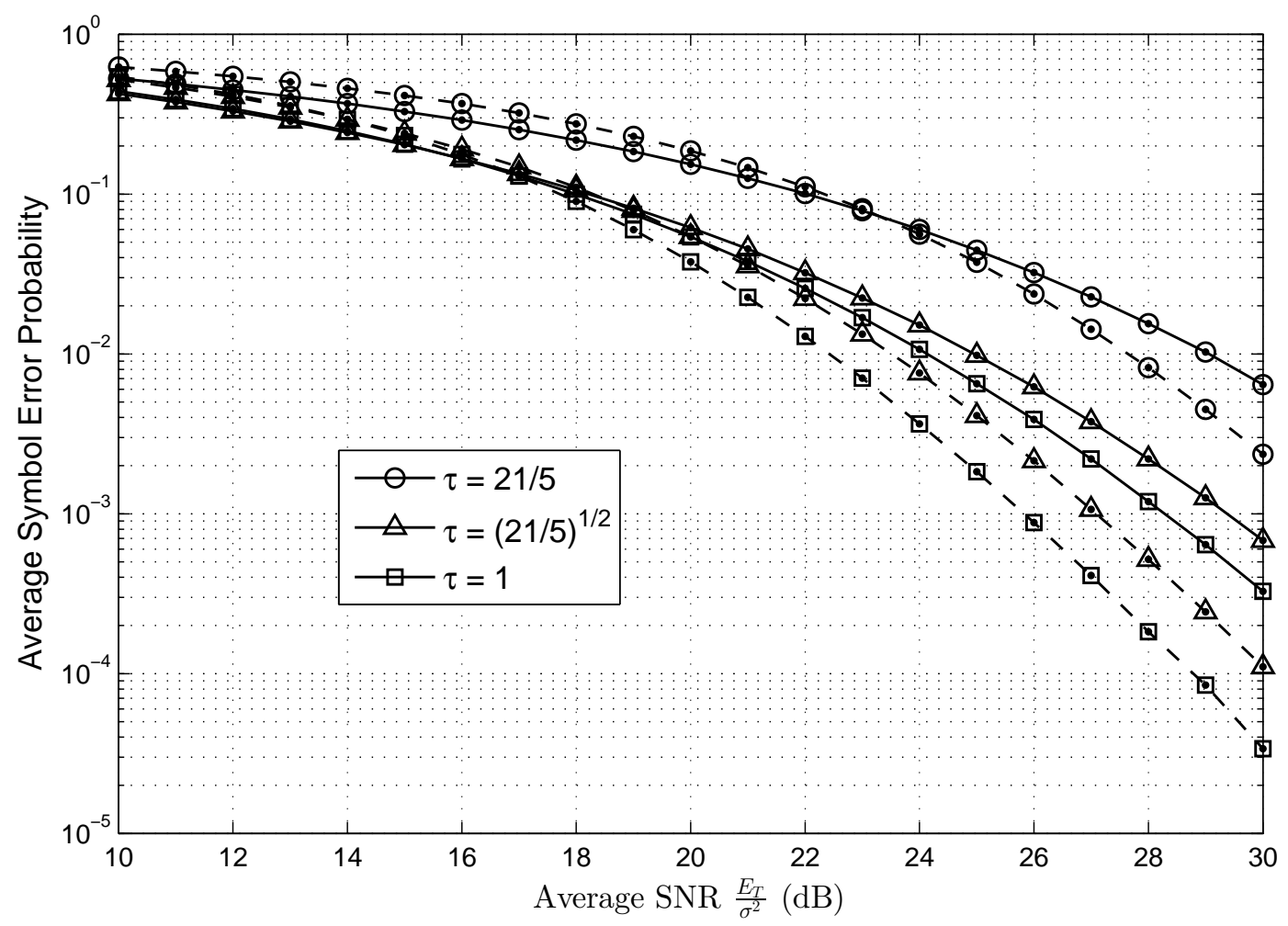

Fig. 3. ASEP of $8 \times 4-\mathrm{QAM}$ over Nakagami-4 fading subject to Laplacian and Gaussian noise. The markers denote simulation results, the solid lines represent the Laplacian noise case, and the dashed lines represent Gaussian noise case. 
TABLE I

Some Special Cases of Generalized Gaussian Distribution

\begin{tabular}{lc}
\hline Noise Distribution & $\boldsymbol{\alpha}$ \\
\hline Impulsive & 0 \\
\hline Gamma & 0.5 \\
\hline Laplacian & 1 \\
\hline Gaussian & 2 \\
\hline Uniform & $\infty$ \\
\hline
\end{tabular}


TABLE II

Some Special Cases of The EGK Distribution

\begin{tabular}{|c|c|c|c|c|}
\hline Envelope Distribution & $m$ & $\xi$ & $m_{s}$ & $\xi_{s}$ \\
\hline \multirow{2}{*}{ Rayleigh } & 1 & 1 & $\infty$ & 1 \\
\hline & $\infty$ & 1 & 1 & 1 \\
\hline \multirow{2}{*}{ Maxwell } & $3 / 2$ & 1 & $\infty$ & 1 \\
\hline & $\infty$ & 1 & $3 / 2$ & 1 \\
\hline \multirow{2}{*}{ Half-Normal } & $1 / 2$ & 1 & $\infty$ & 1 \\
\hline & $\infty$ & 1 & $1 / 2$ & 1 \\
\hline \multirow{2}{*}{ Exponential } & 1 & $1 / 2$ & $\infty$ & 1 \\
\hline & $\infty$ & 1 & 1 & $1 / 2$ \\
\hline \multirow{2}{*}{ Weibull } & 1 & $\xi$ & $\infty$ & 1 \\
\hline & $\infty$ & 1 & 1 & $\xi_{s}$ \\
\hline \multirow{2}{*}{ Nakagami- $m$} & $m$ & 1 & $\infty$ & 1 \\
\hline & $\infty$ & 1 & $m_{s}$ & 1 \\
\hline \multirow{2}{*}{ GNM [13] } & $m$ & $\xi$ & $\infty$ & 1 \\
\hline & $\infty$ & 1 & $m_{s}$ & $\xi_{s}$ \\
\hline \multirow{2}{*}{ Gamma } & $m$ & $1 / 2$ & $\infty$ & 1 \\
\hline & $\infty$ & 1 & $m_{s}$ & $1 / 2$ \\
\hline \multirow{2}{*}{ Generalized Gamma [17], [18] } & $m$ & $\xi / 2$ & $\infty$ & 1 \\
\hline & $\infty$ & 1 & $m_{s}$ & $x i_{s} / 2$ \\
\hline \multirow{2}{*}{ Lognormal } & $\infty$ & 0 & $\infty$ & 1 \\
\hline & $\infty$ & 1 & $\infty$ & 1 \\
\hline \multirow{2}{*}{ Half-Normal-Exponential } & $1 / 2$ & 1 & 1 & 1 \\
\hline & 1 & 1 & $1 / 2$ & 1 \\
\hline \multirow{2}{*}{ Half-Normal-Gamma } & $1 / 2$ & 1 & $m_{s}$ & 1 \\
\hline & 1 & 1 & $1 / 2$ & 1 \\
\hline \multirow{2}{*}{ GNM-Lognormal } & $\infty$ & 0 & $m_{s}$ & $\xi_{s}$ \\
\hline & $m$ & $\xi$ & $\infty$ & 0 \\
\hline \multirow{2}{*}{ Suzuki [19] } & $\infty$ & 0 & 1 & 1 \\
\hline & 1 & 1 & $\infty$ & 0 \\
\hline Rayleigh-Exponential & 1 & 1 & 1 & 1 \\
\hline \multirow{2}{*}{ Maxwell-Lognormal } & $\infty$ & 0 & $3 / 2$ & 1 \\
\hline & $3 / 2$ & 1 & $\infty$ & 0 \\
\hline \multirow{2}{*}{ Maxwell-Exponential } & 1 & 1 & $3 / 2$ & 1 \\
\hline & $3 / 2$ & 1 & 1 & 1 \\
\hline \multirow{2}{*}{ Maxwell-Gamma } & $m$ & 1 & $3 / 2$ & 1 \\
\hline & $3 / 2$ & 1 & $m_{s}$ & 1 \\
\hline \multirow{2}{*}{ Weibull-Lognormal [20] } & $\infty$ & 0 & 1 & $\xi_{s}$ \\
\hline & 1 & $\xi$ & $\infty$ & 0 \\
\hline \multirow{2}{*}{ Weibull-Exponential } & 1 & 1 & 1 & $\xi_{s}$ \\
\hline & 1 & $\xi$ & 1 & 1 \\
\hline Weibull-Weibull & 1 & $\xi$ & 1 & $\xi_{s}$ \\
\hline \multirow{2}{*}{ Weibull-Gamma } & $m$ & 1 & 1 & $\xi_{s}$ \\
\hline & 1 & $\xi$ & $m_{s}$ & 1 \\
\hline Nakaoami-Loonormal & $\infty$ & 0 & $m_{s}$ & 1 \\
\hline 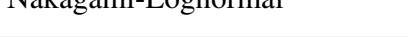 & $m$ & 1 & $\infty$ & 0 \\
\hline$K$ Dictribution & $m$ & 1 & 1 & 1 \\
\hline R-Distribution & 1 & 1 & $m_{s}$ & 1 \\
\hline Generalized-K & $m$ & 1 & $m_{s}$ & 1 \\
\hline GNM-Exnonential & 1 & 1 & $m_{s}$ & $\xi_{s}$ \\
\hline & $m$ & $\xi$ & 1 & 1 \\
\hline GNM-Weibull & $m$ & $\xi$ & 1 & $\xi_{s}$ \\
\hline GNIVI-Weidun & 1 & $\xi$ & $m_{s}$ & $\xi_{s}$ \\
\hline GNM-Gamma & $m$ & 1 & $m_{s}$ & $\xi_{s}$ \\
\hline & $m$ & $\xi$ & $m_{s}$ & 1 \\
\hline
\end{tabular}

